\title{
Ebola fever epidemic 2014: a call for sustainable health and development policies
}

\author{
Steffen Flessa $^{1} \cdot$ Michael Marx ${ }^{2}$
}

Published online: 5 July 2015

(c) Springer-Verlag Berlin Heidelberg 2015

\begin{abstract}
In 2014 an Ebola epidemic emerged in Western Africa (particularly in Guinea, Liberia, Sierra Leone), which with regard to incidence and prevalence exceeded any previous Ebola epidemic [1]. According to estimates of the World Health Organization more than 26,000 people (including suspected cases) suffered from Ebola until April 2015. About $40 \%$ of them died from this infectious disease [2]. The dynamics and intensity of the epidemic took many experts by surprise. Above all, it represented excessive demands on local health care systems as well as-at least initially-of international organizations tasked with coordinated intervention [3]. From a health economic perspective, especially, the complete dysfunctionality of local health care services is not surprising. The Ebola fever epidemic in Western Africa rather reveals fundamental failures in establishing health policies within those countries as well as in development policies of industrialized nations. In the following, some of these structural defects are outlined and conclusions from the Ebola epidemic are drawn.
\end{abstract}

\section{Background}

The Ebola fever is caused by the Ebola virus, leading to a hemorrhagic fever which is fatal in 25-90\% of cases [4]. This disease has been known since 1976, with the first

Steffen Flessa

steffen.flessa@uni-greifswald.de

1 Faculty of Law and Economics, University of Greifswald, Greifswald, Germany

2 Faculty of Heidelberg, University of Heidelberg, Heidelberg, Germany outbreak being described at the river Ebola (Democratic Republic of Congo). Since then, about 35 outbreaks have been registered, with the highest number of cases of one single outbreak so far being 425 (Uganda 2001). Therefore, the epidemic in Western Africa in 2014/15 represents a hitherto completely unknown dimension of Ebola disease [5].

In principle, the transmission of this highly infectious virus is possible via any kind of body fluid. The smallest number of viruses suffices to trigger the disease. Ritual ablutions of the dead, which are common in large parts of Africa, pose a special danger [6]. Until 2014, the outbreaks described were locally restricted and primarily situated in rural areas. They came to a halt after a comparatively short period, being practically self-limiting. Some local societies established traditional rites of quarantine that could be enforced with the rigor of a tribal culture and therefore prevented a further spread of the disease [7].

In the past, repeated outbreaks of Ebola happened. This is primarily due to the fruit bat, persisting in tropical rain forests, being a natural reservoir of the Ebola virus. The eradication of Ebola is thus impossible in the foreseeable future [8].

The Ebola epidemic in 2014/15 stands out significantly from previous epidemics with regard to intensity and dynamics. This is primarily due to the fact that this epidemic expanded to the urban population as well as across borders. On one hand this results in the number of possibly infected people being significantly higher. On the other hand the weakness or absence of social structures in urban settings lowers the social and medical control in case of illness. The previously mentioned funeral rites as well as the completely overburdened and already weak health care systems favored the rapid and massive spread of the disease. The fear of contagion led many health workers to 
leave health service provision which led to an acute shortage of staff in hospitals and health centers. After a short period, local health care systems literally collapsed. Other sectors were severely affected as well. This also resulted in massive declines in the gross national product of the respective countries (estimates differ between US $\$ 6.2$ and 25 billion), in food shortages and the (almost) complete exodus of foreign professionals, also coming from neighboring countries, holding key positions in the economy [9].

Aid for the overburdened countries started pretty delayed in mid-2014, leading to a so far virtually unknown volume of investment in a very short amount of time. In August 2014, the WHO had provided an estimated budget of $\$ 500$ million: in September Ban Ki-Moon called for one billion. To date, the United Nations Mission for Ebola Emergency Response (UNMEER) specified a sum required for emergency aid of \$ 1.5 billion. Indeed, in contrast to other catastrophes, the majority of funds necessary have already been paid.

These amounts are a multiple of national health budgets (Guinea: US \$ 98 million p.a.; Sierra Leone: US \$ 81 million p.a.; Liberia: US \$ 112 million p.a.) [10] and healthrelated development aid, respectively, the affected countries have received in recent years. Whereas financial aid for health care has continuously been reduced or diverted during the past two decades, large amounts of money were suddenly made available. Thereby, Ebola caught up with a group of 'exceptional diseases' which are of international interest, while other equally relevant diseases and health systems needs are often ignored by politics. Over the past decade most funds were allocated to vertical programs to combat three diseases only (malaria, AIDS and TB), while the most significant 'killers' (e.g. diarrhea) are almost completely ignored [11]. Disease-specific programs in particular led to a collapse of community-based education programs, since these only engaged in, for example, bed nets and condoms instead of general health promotion.

This trend was exacerbated by the reduction of professional expertise in the so-called implementing organizations of development cooperation. While medical professionals with specific expertise on the health care systems of countries poor in resources used to be a fixed factor in development assistance, today this expertise is usually purchased externally on short notice. With regard to the health care sector, a stable and consistent development and human resource policy on this basis is only possible to a limited extent.

\section{Ebola fever: a system problem}

Although the treatment of patients does indeed represent a medical challenge of the highest complexity, the epidemic as such is not primarily a medical problem. The intensity and dynamics of dispersal occurs in the context of health care systems that are following a strongly curative strategy, being massively underfunded and heavily influenced by technical and allocative inefficiency. Here the focus is on the chronic shortage of skilled personnel, especially in rural areas. Obsolete practices such as the re-use of inadequately sterilized materials in health services, as well as the transmission in domesticity through unprotected contacts and funeral rites are evidence not only of underfunding, but also of neglecting the education of professionals and the general public. The implementation of Primary Health Care, as declared in 1978 at the World Health Assembly in Alma Ata, could have prevented the massive spread at an earlier stage. In an earlier system approach, primary prevention and basing all health related activities on the local population were identified as key principles. These include education, participation, community work and cooperation with local decision makers [12].

The cooperation with spiritual representatives of religious communities is one example. Since, over the course of many years, health care in the respective countries has been separated completely from the population's spirituality, it is not surprising that changes in funeral rites could have hardly been enforced. These measures were successful only after religious leaders took the floor in allowing an adaptation of rites. Cooperation with religious leaders as well as a long-term work on those rituals would have been possible for years. In this way, the epidemic could most likely have been prevented or at least been restricted to a large extent. In fact, however, health work was often limited to curation. Education, participation and strengthening the self-responsibility of individuals as well as the local community received only little attention.

To date, there is general consensus on sustainable improvement of the health situation only being achievable through consistent strengthening of health systems.

International development cooperation has hardly implemented this insight despite many declarations. Although the budgets of health related development cooperation have increased steadily in recent years, programs progressively concentrated on a few diseases only, and on the development of financial systems. As important as these measures are, unfortunately they cover only partial components of a health system. Community work, as well as education, for example, was neglected inexcusably. As discussed early on, the exceptionality of AIDS, i.e. the deliberate neglect of common criteria of rationality such as effectiveness, efficiency and equity in comparison to other possibilities of resource allocation, lead to a misallocation [13]. Health economists have pointed out the risk of focusing on HIV/AIDS possibly resulting in an increase in 'neglected diseases' [14]. Collateral damage caused by Ebola, e.g. untreated pneumonia, malaria, and declining 
vaccination coverage in the population cannot yet be fully assessed. The massive weakening of the already vulnerable health care systems challenges the affected countries enormously.

The Ebola epidemic points out the need for rational allocation of resources beyond political or media preferences. At the same time there is a risk that Ebola itself is now treated exceptionally, i.e. the measures of combating Ebola are equipped with resources beyond rationality.

\section{Conclusions}

To date, Ebola apparently has a high priority in national and international health and development policies. As important and appropriate as this is, the focus should be on the requirement of not repeating the mistakes of the past. Ebola cannot simply be added to a few target diseases, but the structures have to fundamentally be reconsidered. With regard to the Ebola epidemic in Western Africa in 2014/15, the following requirements derive from a health systems as well as from a health economics perspective:

1. Efficient allocation of resources: The allocation of resources for development cooperation in partnering countries, sectors, regions, diseases and levels of health care (e.g. hospitals versus community-based services) has to be a rational process. For this purpose, objectives and programs of development cooperation need to be defined and operationalized clearly and in the long-term. Exceptions to economic reality lead to a waste of resources and thus to injustice towards the weak and the sick. Given the current situation of highest presence of Ebola in the media, one has to demand that this disease does not become exceptional, but follows the logic of rational resource allocation.

2. Sustainable health and development policies: Development policies of most Western countries conform to long-term objectives deriving from the values of our constitution (e.g. equity, freedom, solidarity). For instance, the Federal Republic of Germany is an advocate of the adoption of Sustainable Development Goals. Consequently, the development policy pursues long-term objectives and promotes the establishment of health care systems being able to function on a longterm basis. In particular, the development of community-based health systems, primary and secondary prevention, as well as participation in, or ownership by the population being considered, has proven to be sustainable. Strengthening primary health services, education, and basic health groups, as well as the training of community health workers, have to be primary goals of development partnership and policies.
In this context the shortage of skilled workers is of paramount importance. Health has to remain a focus of international development cooperation. But the selection of programs and projects should be based on efficiency and effectiveness in the long run, rather than on short-term opinions.

3. Funding: This also implies the choice of funding resources. The tendency towards Global Health Initiatives with highly focused, vertical programs is questionable. Instead, horizontal basic health care systems need to be promoted in order to enable the building of stable societies, the cultivation of health awareness in the population as well as participation of the community and important decision-makers outside of politics. In particular, cooperation with local religious leaders, who are important decision-makers of civil society (such as imams, monks or priests), has to be fostered.

4. Competency in related agencies: The medical, epidemiological and, in particular, health economic expertise of state-funded organizations involved in development cooperation needs to be strengthened in order to establish a long-term health and systems oriented development policy. Access to experts consulted on a short-term basis is essential in most cases. However, it does not represent a complete substitute for in-house expertise.

5. Horizontal programs: The focus on vertical programs combating individual diseases leads to multiple structures, increased costs, inefficiency and inequity. Therefore, strengthening the health care system, if possible including sector-wide but also inter-sectorial programs, has to be promoted. In this way, primary health services can be offered to the whole population comprehensively. The reinforcement of primary and secondary prevention as well as community-based health work is indispensable.

6. Early warning systems and modeling: The intensity and dynamics of the Ebola epidemic took many experts by surprise. This is in part due to the lack of reliable early warning systems and forecasting models for Ebola. In the coming years, the design of such systems has to be carried out not only for Ebola but for other infectious diseases as well. In particular, mathematical models of disease dynamics need to be developed and validated by international groups of experts. Their results should be included regularly in development policies of donor countries. The continuous collection and analysis of solid epidemiological data as well as high-quality system indicators are also essential to early warning systems.

7. Scientific advice: The Scientific Advisory Board of the Federal Ministry for Economic Cooperation and Development was dissolved 5 years ago, as the then 
minister did not see a need for scientific advice. Given the complexity of the Ebola epidemic, one has to call to reinstate this board and to make it a mandatory advisory body. Moreover, the need for implementation research is postulated repeatedly, but little funds are made available for this purpose. In order to provide evidence of the impact of development aid, accompanying operational research is essential.

The Ebola fever epidemic is a wake-up call for higher efficiency, rationality and evidence in the health policies of partner countries and the development policies of donors. If we fail to learn the necessary lessons from the epidemic as well as the attempts at intervention, it can be expected that similar or graver outbreaks of Ebola or other infectious diseases will occur in upcoming years, accompanied by highly negative economic and humane consequences. As health economists we have the methods to accompany the learning process, to develop proposals for rational allocation of resources, to assist in designing the implementation, and to support the evaluation scientifically. Development cooperation should take advantage of this.

\section{References}

1. Benton, A., Dionne, K.Y.: International political economy and the 2014 West African Ebola outbreak. African Stud Rev 58, 223-236 (2015)

2. Cdc (2015): Outbreaks chronology: Ebola hemorrhagic fever. http://www.cdc.gov/vhf/ebola/outbreaks/history/chronology.html. Accessed 4 June 2015
3. De Cock, K.M., Mbori-Ngacha, D., Marum, E.: Shadow on the continent: public health and HIV/AIDS in Africa in the 21st century. Lancet 360, 67-72 (2002)

4. Flessa, S.: Basic health care package without antiretroviral therapy? J Publ Health 16, 145-150 (2008)

5. Leroy, E.M., Kumulungui, B., Pourrut, X., Rouquet, P., Hassanin, A., Yaba, P., Délicat, A., Paweska, J.T., Gonzalez, J.-P., Swanepoel, R.: Fruit bats as reservoirs of Ebola virus. Nature 438, 575-576 (2005)

6. Levin-Sparenberg, E., Gicquelais, R., Blanco, N., Ismail, M.D., Lee, K.H., Foxman, B.: Ebola: the natural and human history of a deadly virus by David Quammen. Am J Epidemiol 181, 151-151 (2015)

7. Nyenswah, T., Blackley, D.J., Freeman, T., Lindblade, K.A., Arzoaquoi, S.K., Mott, J.A., Williams, J.N., Halldin, C.N., Kollie, F., Laney, A.S.: Community quarantine to interrupt Ebola virus transmission-Mawah Village, Bong County, Liberia, AugustOctober, 2014. MMWR 64, 179-182 (2015)

8. Omonzejele, P.F.: Ethical challenges posed by the Ebola virus epidemic in West Africa. J Bioeth Inq 11, 417-420 (2014)

9. Team, W.E.R.: Ebola virus disease in West Africa-the first 9 months of the epidemic and forward projections. N Engl J Med 371, 1481-1495 (2014)

10. WHO: The World Health Report 2008: Primary health carenow more than ever. World Health Organisation, Geneva (2008)

11. WHO (2015a): Ebola situation report, April 2015. http://apps. who.int/ebola/en/ebola-situation-reports. Accessed 4 June 2015

12. WHO (2015b): Neglected tropical diseases. http://www.who.int/ neglected_diseases/diseases/en/. Accessed 4 June 2015

13. World Bank (2015a): Data base. http://data.worldbank.org/indi cator/SH.XPD.TOTL.ZS/countries. Accessed 4 June 2015

14. World Bank (2015b): The economic impact of the 2014 Ebola epidemic: short and medium term estimates for West Africa. https://www.worldbank.org/en/region/afr/publication/the-eco nomic-impact-of-the-2014-ebola-epidemic-short-and-mediumterm-estimates-for-west-africa. Accessed 4 June 2015 